Original Research Article

\title{
Retrospective study on susceptibility and resistance pattern of urinary pathogens in a tertiary care hospital
}

Department of Pharmacology, K.A.P.V. Govt. Medical College, Tiruchirapalli, Tamil Nadu, India

Received: 03 September 2019 Revised: 19 September 2019 Accepted: 20 September 2019

*Correspondence to:

Dr. Sudha Ramu,

Email: sudhapharm71@ gmail.com

Copyright: (C) the author(s), publisher and licensee Medip Academy. This is an openaccess article distributed under the terms of the Creative Commons Attribution NonCommercial License, which permits unrestricted noncommercial use, distribution, and reproduction in any medium, provided the original work is properly cited.

\author{
Sudha Ramu*
}

\begin{abstract}
Background: Indiscriminate and inappropriate use of antimicrobial agents (AMA) resulted in rapid emergence of antimicrobial resistance. Institutional level surveillance program to be carried out to track AMA use. The study was conducted to evaluate the prevalence of uropathogens and their susceptibility and resistance pattern in a tertiary care hospital to revise empirical therapy.

Methods: Urine samples received from the inpatients and outpatients Departments of Mahatma Gandhi memorial hospital for culture sensitivity between January 2018 to December 2018 were included in this study. Data collected from the Department of Microbiology register by using WHONET software. After identification, isolates were tested for antimicrobial susceptibility by the standard Kirby Bauers diffusion method. Descriptive analysis done and results were expressed as percentage.

Results: Out of 3425 samples $68.5 \%$ showed no growth, $15.5 \%$ normal flora and only $15.9 \%$ reported as culture positive. In this study the highest isolate was Escherichia coli (59\%) followed by Klebsiella pneumoniae (10.6\%), Enterococcus sp. (7\%), Staphylococcus aureus (5\%), Candid (3.6\%), Acinetobactor (3\%) and Pseudomonas (2.9\%). Uropathogens developed resistance against penicillins, cephalosporins, macrolides and cotrimaxazole.

Conclusions: This study confirms, the frequently prescribed empirical therapy drugs were less susceptible and developed resistance than less frequently prescribed and costly drugs. The current antimicrobial resistance pattern alarms the irrational and excessive use of antimicrobial agents. Hence the treating physicians should revise empirical therapy periodically based on the institutional antibiogram and resistance pattern reported from the laboratory to preserve antimicrobial source for the future generation.
\end{abstract}

Keywords: Urinary tract infection, Susceptibility and resistance pattern, Antimicrobial agents

\section{INTRODUCTION}

Urinary tract infections (UTI) are one of the most common infections that affect all age groups. Every year about 150 million people are diagnosed with urinary tract infection worldwide. ${ }^{1,2}$ Prevalence of UTI is more common in females than males. Due to the anatomical structure, most of the women approximately $40 \%$ to $50 \%$ will suffer at least one episode of UTI during their lifetime. ${ }^{3}$ High risk factors for UTI are paediatric age groups, elderly patients, patients with spinal cord injuries or catheters, immunocompromised individuals especially HIV, diabetics and congenital urinary tract malformations. ${ }^{4}$
The causative organisms for urinary tract infections are bacteria, predominantly gram negative than gram positive organisms and candida. The prevalence of commonest isolates were Escherichia coli, Klebsiella, Pseudomonas, Staphylococcous and Proteus. ${ }^{5}$ E. coli is the most common causative bacteria being responsible for about 80 percent of infections in acute uncomplicated urinary tract infections. ${ }^{6}$

Antibiotics are the first line of treatment for urinary tract infections, usually patients with symptoms of dysuria and increased frequency of urine are immediately started on empirical therapy. The selection of antibiotic in empirical therapy should be based on information determined from 
the antimicrobial resistance pattern of the urinary pathogens. ${ }^{7}$ The commonly prescribed antimicrobial agents are levofloxacin, ciprofloxacin, nitrofurantoin, cotrimaxazole, nalidixic acid and gentamicin. ${ }^{8}$ Irrational and extensive use of antimicrobial agents has resulted in the development of antimicrobial resistance, which has become a major problem all over the world..$^{9,10}$ UTI increases the morbidity and economical burden of the society. Emergence of multidrug resistance against uropathogens also an important and threatening challenge being faced by the world. ${ }^{10,11}$

The aim of the study was to evaluate the prevalence of uropathogens and their susceptibility and resistance pattern in a tertiary care hospital to guide the clinicians to plan and revise empirical therapy.

\section{METHODS}

This was a retrospective study and the data was collected from the Department of Microbiology laboratory register by using WHONET software. Urine samples received from in patients and outpatients departments of Mahatma Gandhi Memorial hospital attached to K.A.P.V. Government medical college, between January 2018 to December 2018 were included in this study. Samples were processed as per CLSI methodology 2015 guidelines. Smears for Grams staining, bacterial isolates identification and biochemical tests for identifying the species of the pathogens were done. After identification, isolates were tested for antimicrobial susceptibility testing by the standard Kirby Bauers diffusion method. Quality control procedures were incorporated to assure the quality of the media, biochemical and antibiotic discs.

The following standard antibiotic discs were used for the isolates, amikacin (AMK), amoxicillin (AMX), ampicillin (AMP), azithromycin (AZM), cefotaxime (CTX), ceftazidime (CAZ), ceftriaxone (CRO), cephalexin (CEP), chloramphenicol (CHL), ciprofloxacin (CIP), erythromycin (ERY), gentamycin (GEN), gentamycin high (GEH), meropenem (MEM), oxacillin (OXA), penicillin (PEN), piperacillin (PIP), tazobactum (TZP), tigycycline (TCY), tobramycin (TOB), cotrimaxazole (SXT), vancomycin (VAN), doxycycline (DOX), teicoplanin (TEC), cloxacillin (CLO), levofloxacin (LEX), cefoxitin (FOX), cefipime (FEP), ertapenem (ETP), minocycline (MNO), colistin (COL), linezolid (LNX), clindamycin (CLI), imepenam (IPM), cefaperazone sulbactum (CSL), nalidixic acid (NAL), norfloxacin (NOR) and nitrofurantoin (NIT).

Results were entered in Microsoft excel. Descriptive analysis done and results were expressed as percentage.

\section{RESULTS}

Between January 2018 to December 2018, 3425 urine samples were received for culture sensitivity. No growth was reported in 2348 samples and normal flora grown in
531 samples. Numbers of organisms isolated were 546 (Figure 1).

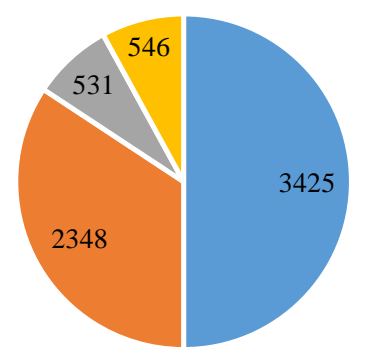

- TOTAL $=$ NO. GRO $=$ NORMAL GROWTH

Figure 1: Distribution of urine samples.

Table 1 shows the prevalence of organisms and the number of isolates.

Table 1: Distribution of bacterial isolates.

\begin{tabular}{|ll|}
\hline Organism & No. of isolates \\
\hline Acinetobacter sp. & 17 \\
\hline Candida sp. & 20 \\
\hline Citrobacter sp. & 5 \\
\hline E. coli & 324 \\
\hline Enterobacter sp. & 9 \\
\hline Enterococcus sp. & 40 \\
\hline Klebsiella sp. & 3 \\
\hline Klebsiella oxytoca & 9 \\
\hline Klebsiella pneumoniae & 58 \\
\hline Pseudomonas aeruginosa & 16 \\
\hline Proteus mirabilis & 7 \\
\hline Pseudomonas sp. & 7 \\
\hline Proteus vulgaris & 2 \\
\hline S. aureus & 29 \\
\hline
\end{tabular}

Highest isolates were E. coli (59\%), Klebsiella Pneumoniae (10.6\%), Enterococcus sp. (7\%), S. Aureus (5\%), Candid (3.6\%), Acinetobactor (3\%) and Pseudomonas (2.9\%).

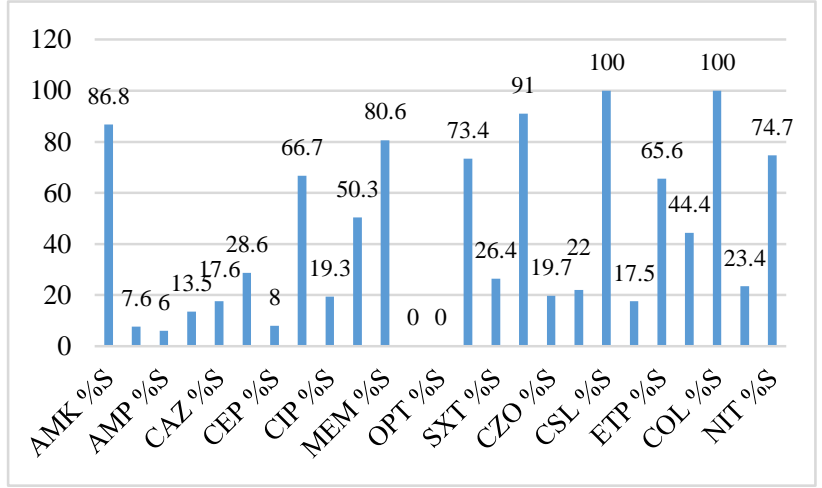

Figure 2: $E$. coli susceptibility pattern. 
E. coli was highly susceptible to (100\%) colistin and cefaperazone sulbactum (CSL), Amikacin (87\%), levofloxacin $(91 \%)$, meropenam $(81 \%)$, tezobactum $(73 \%)$ and nitrofurantoin $(75 \%)$.

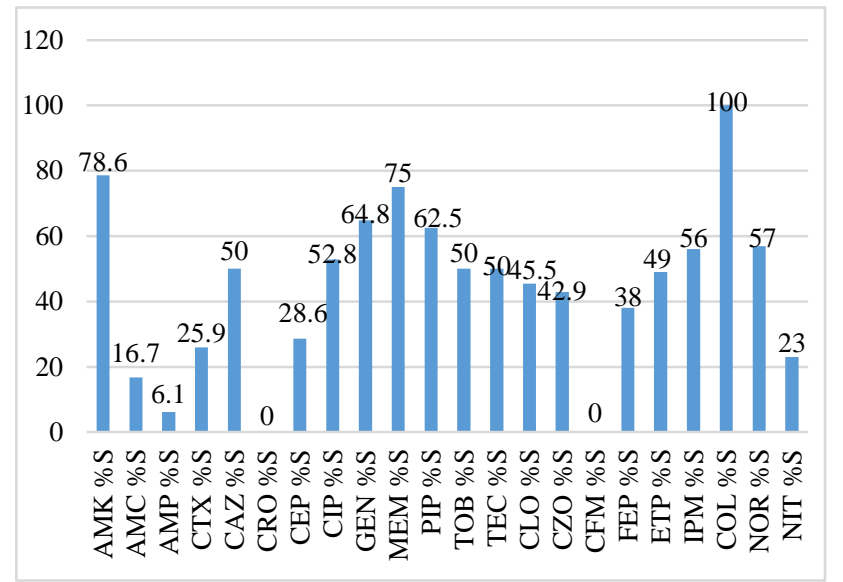

Figure 3: Antimicrobial susceptibility for Klebsiella pneumoniae.

Klebsiella pneumoniae found to have a high susceptiblity to colistin (100\%), amikacin $(79 \%)$ and meropenam $(75 \%)$.

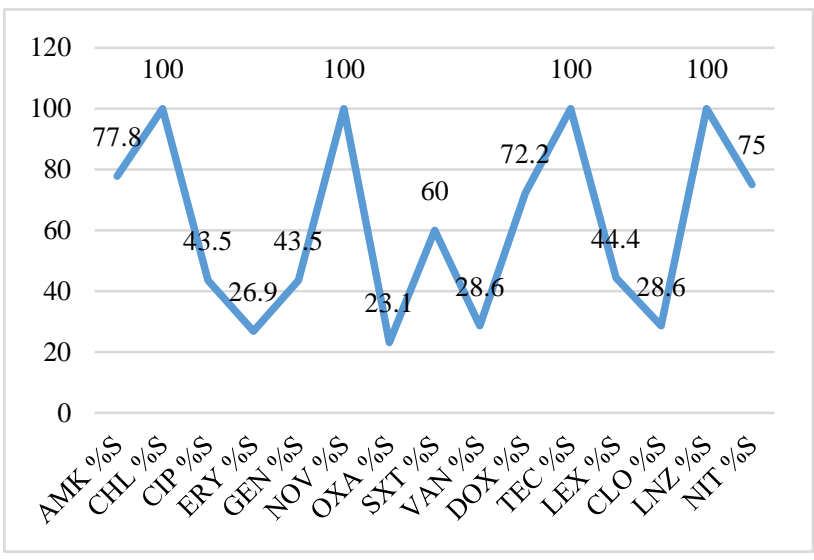

Figure 4: S. aureus susceptibility pattern.

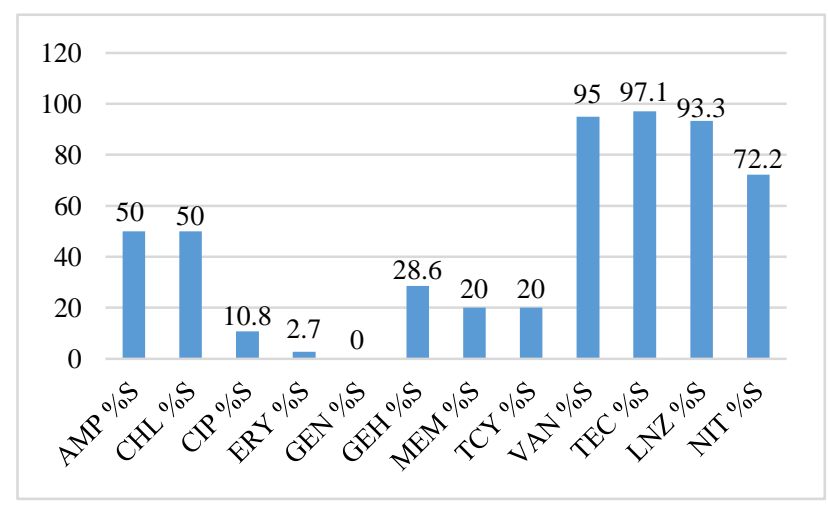

Figure 5: Antimicrobial susceptibility for Enterococcus sp.
S. aureus showed high susceptiblility to chloramphenicol, novobiocin, teicoplanin and linezolid (100\%).

Vancomycin (95\%), teicoplanin (97\%), linezolid (93\%) and nitrofurantoin (72\%) were sensitive against Enterococcus species.

\section{DISCUSSION}

In our study the most common uropathogen reported was E. coli followed by Klebsiella pneumoniae, Enterococcus species, S. Aureus, Candid, Acinetobacter and Pseudomonas. Out of 3425 samples $68.5 \%$ no growth, $15.5 \%$ normal flora and only $15.9 \%$ reported as culture positive.

Various studies from India and other countries also confirms the similar distribution pattern of urinary pathogen. ${ }^{5,6,12,13}$ Obiofu et al study reported S. aureus was the commonest uropathogen in Nigeria. ${ }^{14}$

E. coli was highly susceptible to colistin, cefaperazone sulbactum combination, amikacin, meropenam and levofloxacin. It developed resistance (>80\%) against penicillins (amoxacillin and ampicillin) and all four generations of cephalosporins (cephalexin, cefotaxime, ceftazidime, cefazolin, cefixime and cefipime). Quinolones (nalidixic acid, norfloxacin and ciprofloxacin) and cotrimaxazole $(>70 \%)$ also developed resistance. Gentamicin showed low susceptibility (50\%) to E.coli. Penicillin, cephalosporins, ciprofloxacin and cotrimaxazole resistance to $E$. coli was similar in various studies conducted worldwide. ${ }^{15-18}$

Colistin, amikacin and meropenam were sensitive against Klebsiella and total resistance was seen against penicillins, cephalosporins, quinolones, cotrimaxazole and nitrofurantoin. This findings were coincide with many studies and it was explained, due to the production of extended spectrum beta lactamase (ESBL) by the bacteria its naturally resistant to beta lactam antibiotics. Inappropriate use of AMAs results in emergence of resistance against quinolones, cotrimaxazole and nitrofurantoin. Colistin is not prescribed regularly for its side effects. Amikacin and meropenem are costly drugs. The cost factor of Amikacin and Meropenem restricted the frequent prescription of these drugs and exhibits the high susceptibility. These may be kept as reserve drugs for complications. ${ }^{19,20}$

Enterococcus susceptibility pattern showed vancomycin, teicoplanin, linezolid and nitrofurantoin were highly sensitive. The frequently prescribed drugs penicillins (50\%), quinolones (73\%), erythromycin (84\%) and aminoglycosides $(100 \%)$ were developed resistance. This pattern also comparable to other studies reported in India. $^{21}$ Vancomycin resistance was not seen in our study which is reported in North India. ${ }^{22}$ 
S. aureus was highly susceptible to chloramphenicol, teicoplanin, novobiocin and linezolid. Amikacin, nitrofurantoin and doxycycline were moderately susceptible and total resistance seen against ampicillin and azithromycin. Our study revealed, S. aureus resistance against penicillins, macrolides and low susceptibility to flouroquinolones and aminoglycosides. Onangua et al study also confirms ampicillin resistance, but flouroquinolones are still sensitive in their region. ${ }^{23}$ In contrast to Laksminarayana et al study, S. aureus was highly sensitive to linezolid. ${ }^{24}$ This study also coincides with Toner et al study, which emphasized development of resistance against fluoroquinolone over decade. ${ }^{25}$

\section{CONCLUSION}

Increased availability of antimicrobial agents lead to irrational prescription, excessive use of drugs, rapid development of resistance and depletion of antimicrobial source. Commonly used empirical drugs penicillin, cephalosporins, fluroquinolones and cotrimaxazole were developed resistance, no longer they can be used as empirical therapy. This study showed that colistin, imepenam, amikacin, linezolid and vancomycin continue to the first line of drugs for complications. The current antimicrobial resistance pattern seems to be increase the economical burden of the family and society. Awareness to be created among treating physicians and empirical therapy choice should be revised periodically based on the institutional antibiogram and resistance pattern reported from the laboratory. Our study alarms strict protocol and follow up for antimicrobial use to preserve antimicrobial resource for the future generation.

\section{ACKNOWLEDGEMENTS}

I thank professor and Head of Department of Microbiology for the help and support throughout the study.

Funding: No funding sources

Conflict of interest: None declared

Ethical approval: The study was approved by the Institutional Ethics Committee

\section{REFERENCES}

1. Raju SB, Twari SC. Urinary tract infection-a suitable approach. J Indian Acad Clin Med. 2001;2(4):331-7.

2. Manjunath GN, Prakash R, Vamseedhar Annam KS. Changing trends in the spectrum of antimicrobial drug resistance pattern of uropathogens isolated from hospitals and community patients with urinary tract infections in tumkur and bangalore. Int $\mathrm{J}$ Biol Med Res. 2011;2(2):504-7.

3. Leigh D. Urinary tract infections. In: Smith GR, Easma Charles SF, eds. Topley and Wilsons principles of Bacteriology, virology and immunity, Butler and Tanler Ltd., Frome and London; 1990: 197-214.
4. Foxman B. Epidemiology of urinary tract infections: Inidence, morbidity and economic costs. Dis Mon. 2003;49:53-70.

5. Flores-Mireles AL, Walker JN, Caparon M, Hultgren SJ. Urinary tract infections: epidemiology, mechanisms of infection and treatment options., Nat Rev Microbial. 2015;13(5):269-84.

6. Kothari A, Sagar V. Antibiotic resistance in pathogens causing community-acquired urinary tract infections in India: a multicenter study. The Journal of Infection in Developing Countries. 2008;2(05):354-8.

7. Wilson ML, Gaido L. Laboratory diagnosis of Urinary tract infections in adult patients. Clin Infect Dis. 2004;38:1150-8.

8. Kashef N, Djavid GE, Shahbazi S. Antimicrobial susceptibility patterns of community-acquired uropathogens in Tehran, Iran. J Infection Developing Countries. 2010;4(04):202-6.

9. Kahlmeter G. An international survey of the antimicrobial susceptibility of pathogens from uncomplicated urinary tract infections: the ECO• SENS Project. J Antimicrobial Chemotherap. 2003;51(1):69-76.

10. Acharya A, Gautam R, Subedee L. Uropathogens and their antimicrobial susceptaability pattern in Bharatpur. Nepal Med Coll J. 2011;13(1):30-3.

11. Brown $\mathrm{P}, \mathrm{Ki} \mathrm{M}$, Foxman B. Acute pyelonephritis among adults. Pharmacoeconomics. 2005;23(11):1123-42.

12. Das A, Banergee T. Prevalence of urinary tract infections and susceptibility pattern of uropathogens in women of reproductive age group from North India. J Adv Med. 2015;4(1):5-9.

13. Chaurasia D, Shrivastava RK, Shrivastava S, Dubey D, Songra M. Bacterial pathogens and their antimicrobial susceptibility pattern isolated from urinary tract infection in a tertiary care centre. Int $\mathbf{J}$ Pharma Bio-Sci. 2015;1:20-4.

14. Obiofu EN, Ige OH, Iroro O. Antimicrobial susceptibility pattern of urinary isolates from outpatients suspected for urinary tract infection. GSC Biol Pharm Sci. 2018;5(3):1-11.

15. Shanthi B, Selvi R, Madhumathy A. Antimicrobial Susceptibility Pattern of Escherichia coli from Patients with Urinary Tract Infections in a Tertiary Care Hospital. Int J Curr Microbiol App Sci. 2018;7(1):289-94.

16. Chittagong B. A study of antibacterial susceptibility and resistance pattern of E. coli causing urinary tract infection in Chittagong, Bangladesh. Asian J Biol Sci. 2011;4(7):548-55.

17. Kibret M, Abera B. Antimicrobial susceptibility pattern of E.coli from clinical sources in Northeast Ethiopia. Afr Health Sci. 2011;11(1):40-5.

18. De Francesco MA, Ravizzola G, Peroni L, Negrini R, Manca N. Urinary tract infections in Brescia, Italy: etiology of uropathogens and antimicrobial resistance of common uropathogens. Med Sci Monitor. 2007;13(6):136-44. 
19. Varghese A, George S, Gopalakrishnan R, Mathew A. Antibiotic susceptibility pattern of Klebsiella pneumoniae isolated from cases of urinary tract infection in a tertiary care setup. J Evol Med Dent Sci. 2016;5(29):1470-4.

20. Ullah F, Malik S, Ahmed J. Antibiotic susceptibility pattern and ESBL prevalence in nosocomial Escherichia coli from urinary tract infections in Pakistan. Afr J Biotech. 2009;8(16):3921-6.

21. Bharti AK, Farooq U, Singh S, Kaur N, Ahmed R, Singh K. Incidence of Enterococcal Urinary Tract Infection and it's Sensitivity Pattern among Patients Attending Teerthanker Mahaveer Medical College and Research Centre, Moradabad, India. Int J Sci Study. 2016;3(12):112-6.

22. Goel V, Kumar D, Kumar R, Mathur P, Singh S. Community acquired enterococcal urinary tract infections and antibiotic resistance profile in North India. J Lab Phy. 2016;8(1):50.
23. Onanuga A Awhowho GO. Antimicrobial resistance of staphylococcus aureus strains from patients with urinary tract infections in Yenagoa, Nigeria. J Pharm Bioallied Sci. 2012;4(3):226-30.

24. Lakshminarayana SA, Chavan SKD, Prakash R, Sangeetha $\mathrm{S}$. Bacterial pathogens in urinary tract infection and antibiotic susceptibility pattern from a teaching hospital, Bangaluru, India. Int $\mathbf{J}$ Curr Microbial App Sci. 2015;4(11):731-6.

25. Toner L, Papa N, Perera M, Aliyu SH, Dev H, Lawrentschuk N, et al. Staphylococcus aureus urinary tract bacteriuria: single-institutional antibiotic susceptibility trends over a decade. Bladder. 2017;4(3):30

Cite this article as: Ramu S. Retrospective study on susceptibility and resistance pattern of urinary pathogens in a tertiary care hospital. Int J Basic Clin Pharmacol 2019;8:2211-5. 